\title{
A probability theoretical access to extragalactic microlensing
}

\author{
B. Neindorf ${ }^{\star}$ \\ Hamburger Sternwarte, University of Hamburg, Gojenbergsweg 112, 21029 Hamburg, Germany \\ Received 15 October 2002 / Accepted 14 January 2003

\begin{abstract}
A probability theoretical method is presented which enables the calculation of microlensing autocorrelation functions. Following an idea of Deguchi and Watson the Holtsmark-Markov method is employed to reduce this calculation to the numerical evaluation of twodimensional integrals. Comparing these results with autocorrelation functions calculated from measurements, consistent preliminary information about the individual lens system B1600+434 has already been obtained.
\end{abstract}

Key words. methods: numerical - gravitational lensing - microlensing - quasars: individual: B1600+434

\section{Introduction}

Microlensing in multiple imaged quasars that is produced by stars and other compact objects in the lensing galaxy commonly is regarded as a nuisance for the determination of the time delay between different macroimages of the quasar. On the other hand the variability of the light curves caused by microlensing provides additional information about the source and the lens, whereby in this context the signal is seen to be contaminated by the intrinsic variability of the quasar.

A lot of different methods have been employed to extract the information added to the detected signal by microlensing. Analysis of individual high magnification events in the light curves has been done for example by Grieger et al. (1988, 1991) using ray shooting or by Shalyapin (2001) and Yonehara (2001) based on the observations of the quadruple quasar QSO 2237+0305 (Einstein Cross) by Woźniak et al. (2000).

More effort has been put into the statistical analysis of light curves. Besides the statistics of high amplification events (Witt et al. 1993; Wyithe et al. 2000b) estimates for the source radius and the mass spectrum of the lens from light curves have been of special interest (Refsdal \& Stabell 1993, 1997; Schmidt \& Wambsganss 1998; Wyithe et al. 2000a; Refsdal et al. 2000). Inverse ray shooting (Kayser et al. 1986; Schneider \& Weiss 1986) refined by the inclusion of the hierarchical tree code developed by Wambsganss (1990) or an image tracking method to solve the lens equation that has been developed independently by Lewis et al. (1993) and Witt (1993) have been the preferred workhorses for the calculation of sample light curves from numerical simulations. Wambsganss et al. (1990) already calculated autocorrelation functions using ray shooting. To get reliable estimates for the variance or the autocorrelation function given the underlying microlensing model it is essential to evaluate large statistically independent samples of those artificial light curves. Especially for the determination of an

\footnotetext{
* e-mail: b.neindorf@hamburg.de
}

autocorrelation function the random starfield has to be taken large enough to eliminate the influence from the finite length of the track. This turns out to be a time consuming task. Additionally all results are subject to statistical fluctuations that have to be controlled by relative error estimates.

Application of probability theory to microlensing enables the calculation of expectation values and removes the dependence of the theoretical modelling on statistical uncertainty due to finite light curve lengths and individual realisations of the random star field. Deguchi \& Watson (1987) calculated the variance of light curves caused by microlensing. They employed the Holtsmark-Markov method that has been presented by Chandrasekhar (1943) in the context of the calculation of the gravitational force resulting from a random star field. This approach has been generalized by Seitz \& Schneider (1994) and Seitz et al. (1994) to include the determination of the light curve's autocorrelation function. Unfortunately this project has not been followed further, mainly because no means has been found to reduce the numerical effort to a reasonable level. Their final result was given as a three dimensional integral plus a series expansion that had to be evaluated numerically. As an additional complication the integrand of this function was given by a three dimensional integral plus series expansion too, so practical application of this method turned out to be rather cumbersome.

This paper presents a modified approach to this problem, that results in an expression for the autocorrelation function that can be evaluated by a twodimensional numerical integration which can be done in a couple of minutes on a personal computer. A correlation function for the light deflection in the lens is part of every integral and it has been evaluated by a onedimensional numerical integration once and for all and the results have been tabulated for future use.

Results of model calculations have been compared to autocorrelation functions estimated from the $8.5 \mathrm{GHz}$ VLA radio data of the double quasar B1600+434 (Koopmans et al. 2000). 
The obtained system parameters came out to be of the same order as estimates by Koopmans and de Bruyn (2000).

\section{Basic theory}

A microlensing situation can be described in the context of a thin lens approximation (Schneider et al. 1992) by the normalized lens equation (Paczyński 1986; Kayser et al. 1986)

$\zeta=J_{0} z-\operatorname{sgn} \kappa \sum_{i} m_{i} \frac{z-z_{i}}{\left|z-z_{i}\right|^{2}}$

using the shorthand notation

$J_{0}:=\left(\begin{array}{cc}1+\gamma & 0 \\ 0 & 1-\gamma\end{array}\right)$

As is common practice in microlensing theory, image locations $z$ and star positions $z_{i}$ in the lens plane are given in units of the Einstein radius

$z_{0}:=\sqrt{\frac{M_{0}}{\pi \Sigma_{\mathrm{cr}}\left|1-\kappa_{\mathrm{c}}\right|}}$,

where $\Sigma_{\text {cr }}$ is the critical surface mass density $\Sigma_{\mathrm{cr}}:=\frac{c^{2}}{4 \pi G D}$ and $D$ an effective distance expressed by angular size distances $D:=\frac{D_{\mathrm{ds}} D_{\mathrm{d}}}{D_{\mathrm{s}}}$. Vectors $\zeta$ in the source plane are given in units of $\zeta_{0}:=z_{0}\left(1-\kappa_{\mathrm{c}}\right) D_{\mathrm{s}} / D_{\mathrm{d}}$ and the masses $m_{i}$ of the individual stars are normalized by the unit mass $M_{0}$ which is conventionally but not necessarily taken as a solar mass. A surface mass density $\Sigma$ is defined by a projection of the mass between source and observer onto the lens plane.

If for a multiple quasar the normalized mass density $\kappa_{\mathrm{t}}:=$ $\Sigma / \Sigma_{\text {cr }}$ and the shear $\gamma^{\prime}$ are given by a macrolens model at each position of a macroimage with $\kappa_{\mathrm{t}}=\kappa_{\mathrm{c}}+\kappa_{\mathrm{s}}$, where $\kappa_{\mathrm{c}}$ represents the continuum mass density and $\kappa_{\mathrm{s}}$ the smeared out normalized surface mass density in stars and other compact objects, then

$\gamma:=\frac{\gamma^{\prime}}{1-\kappa_{\mathrm{c}}}$ and $\kappa:=\frac{\kappa_{\mathrm{S}}}{1-\kappa_{\mathrm{c}}}$

are the normalized shear and surface mass density in stars respectively. The function

$S(z)=\sum_{i} S_{i}(z)$ where $S_{i}(z)=m_{i} \frac{z-z_{i}}{\left|z-z_{i}\right|^{2}}$

describes the effect of the stars. The amplification $\mu$ of a point source caused by microlensing at $\zeta$ can be expressed by an integral in the lens plane

$\mu(\zeta)=\frac{1}{\left|1-\kappa_{\mathrm{c}}\right|^{2}} \int_{\mathcal{L}} \delta\left(\zeta-J_{0} z+\operatorname{sgn} \kappa S(z)\right) \mathrm{d}^{2} z$.

The flux density of an extended source measured by an observer is simply the convolution of the magnification with the intrinsic brightness profile

$I=\int_{\mathcal{S}} \mu(\zeta) I(\zeta) \mathrm{d}^{2} \zeta$

Since there is no specific information available about the stars in the lensing galaxy a probabilistic approach will be considered. The number of stars $N$, their masses $m_{i}$ and positions $z_{i}$ can be treated as random variables when calculating the expectation values of physical quantities. The mean flux density

$\langle I\rangle=\frac{1}{\left|1-\kappa_{\mathrm{c}}\right|^{2}\left[(1-\kappa)^{2}-\gamma^{2}\right]} \int_{\mathcal{S}} \mathcal{I}(\zeta) \mathrm{d}^{2} \zeta$

is not suitable for the analysis of microlensing since it does only depend on the mean mass density and not on it's distribution. A quantity that characterizes the flux density fluctuations and does depend on the mass distribution in the lens is the expectation value of the square of the flux density $\left\langle I^{2}\right\rangle$ that has been considered by Deguchi \& Watson (1987). As was demonstrated by Seitz \& Schneider (1994) there is little extra effort needed for the calculation of the expectation value of the flux density product at nonzero time lag $\left\langle I\left(t_{1}\right) I\left(t_{2}\right)\right\rangle$ which measures the correlation of fluctuations in time.

The easiest and possibly only way to derive and calculate these expectation values assumes an extended source with Gaussian profile

$\mathcal{I}(\zeta)=\frac{\mathcal{I}_{0}}{\pi R^{2}} \mathrm{e}^{-\frac{\left(\zeta-\zeta_{0}\right)^{2}}{R^{2}}}$.

Besides smoothing the fluctuations as is to be expected with extended sources, this choice of brightness profile forces convergence of the upcoming integral transformations and removes edge effects under coordinate transformations. Another restricting preliminary in the course of this project is the assumption that the lens is static and all variability stems from the relative motion of the source and the lens as a whole, a restriction that can be justified by the findings of Wambsganss \& Kundic (1993) or Wyithe et al. (2000a), who show how relative motion with respect to the lens can be approximated for statistical purposes by an equivalent effective bulk velocity. Under these preliminaries the expected flux density product is

$\left\langle I\left(\zeta_{01}\right) I\left(\zeta_{02}\right)\right\rangle=\int_{\mathcal{S}^{2}}\left\langle\mu\left(\zeta_{1}\right) \mu\left(\zeta_{2}\right)\right\rangle \mathcal{I}\left(\boldsymbol{\zeta}_{1}\right) \mathcal{I}\left(\zeta_{2}\right) \mathrm{d}^{2} \zeta_{1} \mathrm{~d}^{2} \zeta_{2}$

and the mean amplification product is given by

$\left\langle\mu\left(\zeta_{1}\right) \mu\left(\zeta_{2}\right)\right\rangle=$

$$
\int_{\mathcal{R}^{4}} \phi\left(\boldsymbol{S}_{1}, \boldsymbol{S}_{2}\right) \prod_{j=1}^{2} \delta\left(\zeta_{j}-J_{0} z_{j}+\operatorname{sgn} \kappa \boldsymbol{S}_{j}\right) \frac{\mathrm{d}^{2} S_{j} \mathrm{~d}^{2} z_{j}}{\left|1-\kappa_{\mathrm{c}}\right|^{4}},
$$

where $\boldsymbol{S}_{j}:=\boldsymbol{S}\left(z_{j}\right)$ and $\phi\left(\boldsymbol{S}_{1}, \boldsymbol{S}_{2}\right)$ is the probability density for the correlated light deflection at the positions $z_{1}, z_{2}$ in the lens plane. Expressing $\phi\left(\boldsymbol{S}_{1}, \boldsymbol{S}_{2}\right)$ by its Fourier transform (also called characteristic function in probability theory)

$\phi\left(\boldsymbol{S}_{1}, \boldsymbol{S}_{2}\right)=$

$$
\frac{1}{(2 \pi)^{4}} \int_{\mathcal{R}^{4}} Q_{2}\left(\tau_{1}, z_{1}, \tau_{2}, z_{2}\right) \mathrm{e}^{-i\left(\tau_{1} S_{1}+\tau_{2} S_{2}\right)} \mathrm{d}^{2} \tau_{1} \mathrm{~d}^{2} \tau_{2},
$$

the $\delta$-functions can be evaluated by integrating over the deflection parameters $\mathrm{d}^{2} S_{1}, \mathrm{~d}^{2} S_{2}$. The double convolution of Eq. (10) then decouples into two distinct Fourier transforms of the source profile, and after dropping the additional subscript specifying the source centre, the result can be written as follows

$$
\begin{aligned}
& \left\langle I\left(\zeta_{1}\right) I\left(\zeta_{2}\right)\right\rangle=\frac{\mathcal{I}_{0}^{2}}{(2 \pi)^{4}\left|1-\kappa_{\mathrm{c}}\right|^{4}} \int_{\mathcal{R}^{8}} Q_{2}\left(\boldsymbol{\tau}_{1}, z_{1}, \tau_{2}, z_{2}\right) \\
& \quad \times \prod_{j=1}^{2} \exp \left(-\frac{\tau_{j}^{2} R^{2}}{4}+i \operatorname{sgn} \kappa \tau_{j}\left(\zeta_{j}-J_{0} z_{j}\right)\right) \mathrm{d}^{2} \tau_{j} \mathrm{~d}^{2} z_{j} .
\end{aligned}
$$


Before this course can be followed further, the characteristic function $Q_{2}\left(\tau_{1}, z_{1}, \tau_{2}, z_{2}\right)$ for the correlated light deflection has to be derived.

\section{The characteristic function of the deflection probability}

The calculation of the probability density of a sum of several independent random variables by means of the HoltsmarkMarkow method has already been described extensively by Chandrasekhar (1943) in the context of the estimation of the gravitational force excerted by a random star field. The main idea is based on the fact that the probability density of a sum of $N$ independent random variables can be expressed by a $(N-1)$-fold convolution of the individual probability densities. A Fourier transformation converts this into a $N$-fold product of the individual Fourier transforms. If the probability densities of all the $N$ random variables are equal, so will be their Fourier transforms, and the probability density of the sum can then be found by inverse Fourier transformation of the $N$ th power of the probability density for the deflection by a single star.

The probability density for the correlated light deflection can be decomposed to give

$\phi\left(\boldsymbol{S}_{1}, \boldsymbol{S}_{2}\right)=\sum_{N=0}^{\infty} P(N, \mathcal{L}) \phi_{N}\left(\boldsymbol{S}_{1}, \boldsymbol{S}_{2}\right)$

where $P(N, \mathcal{L})$ is the probability to find exactly $N$ stars in the lens $\mathcal{L}$ and $\phi_{N}\left(\boldsymbol{S}_{1}, \boldsymbol{S}_{2}\right)$ is the conditional probability for the light deflection given the number of stars $N$.

The number of stars in the lens will be taken Poissondistributed with mean $\langle N(\mathcal{L})\rangle=n\|\mathcal{L}\|$

$P(N, \mathcal{L}):=\mathrm{e}^{-\langle N(\mathcal{L})\rangle} \frac{\langle N(\mathcal{L})\rangle^{N}}{N !}$

where $\|\mathcal{L}\|$ is the area of the lens and $n$ the number density of stars

$n:=\frac{|\kappa|}{\pi\langle m\rangle}$.

Comparison with the findings of Seitz \& Schneider (1994) shows that the assumption about the number of stars is not crucial because they get to the same result by taking the limit of $\phi_{N}\left(\boldsymbol{S}_{1}, \boldsymbol{S}_{2}\right)$ when $N \rightarrow \infty$. The conditional probability for the light deflection given the number of stars will be

$$
\begin{aligned}
& \phi_{N}\left(\boldsymbol{S}_{1}, \boldsymbol{S}_{2}\right)= \\
& \int_{\mathcal{L}^{2 N}} \prod_{j=1}^{2} \delta\left(\boldsymbol{S}_{j}-\sum_{k=1}^{N} \boldsymbol{S}_{j k}\right) \prod_{i=1}^{N} \phi\left(\boldsymbol{S}_{1 i}, \boldsymbol{S}_{2 i}\right) \mathrm{d}^{2} \boldsymbol{S}_{1 i} \mathrm{~d}^{2} \boldsymbol{S}_{2 i},
\end{aligned}
$$

where $S_{j i} \equiv S_{i}\left(z_{j}\right)$ is shorthand for the contribution of the $i$ th star to the deflection angle at the position $z_{j}$ in the lens plane. When the stars are taken to be identical distributed with probability density

$\phi\left(z_{i}\right)=\|\mathcal{L}\|^{-1}$

for the position vector, all correlations between the stars will be neglected. The mass distribution characterized by it's probability density $\phi\left(m_{i}\right)$ will be left undetermined at this stage.
The probability density for the correlated deflection by a single star can then be written as

$\phi\left(\boldsymbol{S}_{1 i}, \boldsymbol{S}_{2 i}\right)=\int_{0}^{\infty} \int_{\mathcal{L}} \prod_{j=1}^{2} \delta\left(\boldsymbol{S}_{j i}-m_{i} \frac{\boldsymbol{z}_{j}-\boldsymbol{z}_{i}}{\left|\boldsymbol{z}_{j}-\boldsymbol{z}_{i}\right|^{2}}\right) \frac{\mathrm{d}^{2} z_{i}}{\|\mathcal{L}\|} \phi\left(m_{i}\right) \mathrm{d} m_{i}(19)$

where the contribution of each star to the light deflection is averaged for position and mass of this star.

Fourier transforming this equation gives the characteristic function for this probability density

$$
\begin{aligned}
p\left(\boldsymbol{\tau}_{1}, \boldsymbol{z}_{1}, \boldsymbol{\tau}_{2}, \boldsymbol{z}_{2}\right) & =\int_{\mathcal{R}^{4}} \phi\left(\boldsymbol{S}_{1 i}, \boldsymbol{S}_{2 i}\right) \mathrm{e}^{i\left(\tau_{1} \boldsymbol{S}_{1 i}+\tau_{2} S_{2 i}\right)} \mathrm{d}^{2} S_{1 i} \mathrm{~d}^{2} S_{2 i} \\
& \left.=\int_{0}^{\infty} \int_{\mathcal{L}} \mathrm{e}^{i m\left(\tau_{1} \frac{z_{1}-z}{\left|z_{1}-z\right|^{2}}+\tau_{2} \frac{z_{2}-z}{\left|z_{2}-z\right|^{2}}\right.}\right) \frac{\mathrm{d}^{2} z}{\|\mathcal{L}\|} \phi(m) \mathrm{d} m,
\end{aligned}
$$

so the Fourier transform of Eq. (17) results in

$$
\int_{\mathcal{R}^{4}} \phi_{N}\left(\boldsymbol{S}_{1}, \boldsymbol{S}_{2}\right) \mathrm{e}^{i\left(\tau_{1} S_{1}+\tau_{2} S_{2}\right)} \mathrm{d}^{2} S_{1} \mathrm{~d}^{2} S_{2}=p^{N}\left(\boldsymbol{\tau}_{1}, \boldsymbol{z}_{1}, \boldsymbol{\tau}_{2}, \boldsymbol{z}_{2}\right) .
$$

Following this simplification the expectation value with respect to the number of stars involved Eq. (14) can be evaluated:

$$
\begin{aligned}
Q_{2}\left(\boldsymbol{\tau}_{1}, \boldsymbol{z}_{1}, \boldsymbol{\tau}_{2}, \boldsymbol{z}_{2}\right) & =\sum_{N=0}^{\infty} P(N, \mathcal{L}) p^{N}\left(\boldsymbol{\tau}_{1}, z_{1}, \boldsymbol{\tau}_{2}, \boldsymbol{z}_{2}\right) \\
& =\mathrm{e}^{-\langle N(\mathcal{L})\rangle\left[p\left(\boldsymbol{\tau}_{1}, z_{1}, \boldsymbol{\tau}_{2}, z_{2}\right)-1\right]} \\
& \left.=\mathrm{e}^{n \int_{0}^{\infty} \int_{\mathcal{L}}\left[\mathrm{e}^{i m\left(\tau_{1} \frac{z_{1}-z}{\left|z_{1}-z\right|^{2}}+\tau_{2}\right.} \frac{z_{2}-z}{\left|z_{2}-z\right|^{2}}\right)}-1\right] \mathrm{d}^{2} z \phi(m) \mathrm{d} m
\end{aligned}
$$

After transformation to central and relative coordinates

$$
\begin{aligned}
& T:=\frac{\tau_{1}+\tau_{2}}{2}, \quad t:=\tau_{2}-\tau_{1} \\
& R_{\mathrm{c}}:=\frac{z_{1}+z_{2}}{2}, \rho:=z_{2}-z_{1}
\end{aligned}
$$

the limit for an infinite lens can be taken

$$
\begin{array}{r}
Q_{2}\left(\boldsymbol{\tau}_{1}, \boldsymbol{z}_{1}, \boldsymbol{\tau}_{2}, \boldsymbol{z}_{2}\right)=Q_{2}\left(\boldsymbol{T}-\frac{\boldsymbol{t}}{2}, \boldsymbol{R}_{\mathrm{c}}-\frac{\boldsymbol{\rho}}{2}, \boldsymbol{T}+\frac{\boldsymbol{t}}{2}, \boldsymbol{R}_{\mathrm{c}}+\frac{\boldsymbol{\rho}}{2}\right) \\
=Q_{2}\left(\boldsymbol{T}-\frac{\boldsymbol{t}}{2},-\frac{\boldsymbol{\rho}}{2}, \boldsymbol{T}+\frac{\boldsymbol{t}}{2}, \frac{\boldsymbol{\rho}}{2}\right) \mathrm{e}^{2 i n \pi\langle m\rangle \boldsymbol{T} \boldsymbol{R}_{\mathrm{c}}}+O\left(\|\mathcal{L}\|^{-1}\right) .
\end{array}
$$

Performing the same coordinate transformation in Eq. (13), the $\mathrm{d}^{2} R_{\mathrm{c}}$ integral can be evaluated and the result will be proportional to $\delta(\boldsymbol{T})$, therefore in this context it suffices to explicitely calculate

$$
\begin{aligned}
& Q(\boldsymbol{t}, \boldsymbol{\rho}):=Q_{2}\left(-\frac{\boldsymbol{t}}{2},-\frac{\boldsymbol{\rho}}{2}, \frac{\boldsymbol{t}}{2}, \frac{\boldsymbol{\rho}}{2}\right)= \\
& \quad \exp \left(n \int_{0}^{\infty} \int_{\mathcal{L}}\left[\mathrm{e}^{i m t\left(\frac{\rho-z}{|\boldsymbol{\rho}-z|^{2}}+\frac{\rho+z}{|\boldsymbol{\rho + z}|^{2}}\right)}-1\right] \mathrm{d}^{2} z \phi(m) \mathrm{d} m\right) .
\end{aligned}
$$

Further simplification can be achieved by the following coordinate transformation where $\left[\tau_{j x}, \tau_{j y}\right]^{T} \equiv \tau_{j}$,

$\boldsymbol{r}(\boldsymbol{z}):=\sum_{j=1}^{2}\left(\begin{array}{cc}\tau_{j x} & \tau_{j y} \\ \tau_{j y} & -\tau_{j x}\end{array}\right) \frac{\boldsymbol{z}_{j}-\boldsymbol{z}}{\left|\boldsymbol{z}_{j}-\boldsymbol{z}\right|^{2}}$ 
which is especially easy to handle in complex notation $\boldsymbol{r}:=$ $\left(\begin{array}{l}x \\ y\end{array}\right) \rightarrow x+i y$ and $\tau_{j} \rightarrow \tau_{j x}+i \tau_{j y}:$

$$
\begin{aligned}
r(z) & =\sum_{j=1}^{2} \frac{\tau_{j}}{z_{j}-z} \\
& =\frac{T+\frac{t}{2}}{R_{\mathrm{c}}-z+\frac{\rho}{2}}+\frac{T-\frac{t}{2}}{R_{\mathrm{c}}-z-\frac{\rho}{2}} \\
& =\frac{2 T\left(R_{\mathrm{c}}-z\right)-\frac{t \rho}{2}}{\left(R_{\mathrm{c}}-z\right)^{2}-\left(\frac{\rho}{2}\right)^{2}} .
\end{aligned}
$$

The $x$-component or real part of $r$ is equal to the exponent of the integral in Eq. (22) and the inverse mapping is

$$
z(r)=R_{\mathrm{c}}-\frac{T}{r} \pm \sqrt{\left(\frac{T}{r}\right)^{2}-\frac{t \rho}{2 r}+\left(\frac{\rho}{2}\right)^{2}}
$$

For a completion of this transform the Jacobi determinant of the mapping $z \rightarrow r$ is needed. For the inverse mapping the determinant reads

$\left|\frac{\partial \boldsymbol{r}}{\partial z}\right|=\left|\sum_{j=1}^{2} \frac{\tau_{j}}{\left(z_{j}-z\right)^{2}}\right|^{2}$.

Further simplification arises with the restriction to $\boldsymbol{R}_{\mathrm{c}}=0$ and $\boldsymbol{T}=0$, when

$z(r)= \pm \frac{\rho}{2 \sqrt{r}} \sqrt{r-2 s}$

defining the vector $s$ by the mapping

$s:=\frac{t}{\rho} \Longleftrightarrow s=\left(\begin{array}{cc}t_{x} & t_{y} \\ t_{y} & -t_{x}\end{array}\right) \frac{\rho}{|\rho|^{2}}=s\left(\begin{array}{c}\cos \chi \\ \sin \chi\end{array}\right)$.

The Jacobi determinant reduces to

$\left|\frac{\partial \boldsymbol{r}}{\partial z}\right|_{T=0}=\frac{4 r^{3}}{t^{2}}|\boldsymbol{r}-2 \boldsymbol{s}|$,

which can easily be inverted for the employment of the coordinate transformation in Eq. (25).

This transformation then results in

$$
\begin{aligned}
Q(\boldsymbol{t}, \boldsymbol{\rho}) & =\exp \left[2 n t^{2} \int_{0}^{\infty} \int_{\mathcal{R}^{2}} \frac{\mathrm{e}^{i m x}-1}{4 r^{2}} \frac{\mathrm{d}^{2} r}{|\boldsymbol{r}-2 \boldsymbol{s}|} \phi(m) \mathrm{d} m\right] \\
& =\exp \left[-\frac{n}{2} \rho^{2} a(\boldsymbol{s})\right]
\end{aligned}
$$

where further task has been reduced to the determination of the complex function $a(s)$ or, after seperating out the evaluation of the mass spectrum via

$a(\boldsymbol{s})=\int_{0}^{\infty} \alpha(m, \boldsymbol{s}) \phi(m) \mathrm{d} m$,

to the determination of

$$
\alpha(m, s)=s^{2} \int_{\mathcal{R}^{2}} \frac{1-\mathrm{e}^{i m x}}{r^{3}} \frac{\mathrm{d}^{2} r}{|\boldsymbol{r}-2 \boldsymbol{s}|} .
$$

A major progress has been achieved because in Cartesian coordinates the part of the integral not involving the exponential function can be evaluated seperately

$\alpha(m, s)=\int_{-\infty}^{\infty} \frac{1-\mathrm{e}^{i m s x}}{x^{2}} f(x, \chi) \mathrm{d} x$,

where

$f(x, \chi):=\int_{-\infty}^{\infty} \frac{x^{2} \mathrm{~d} y}{r^{3} \sqrt{(x-2 \cos \chi)^{2}+(y-2 \sin \chi)^{2}}}$

can be transformed into an elliptic integral for further evaluation. Substitution of

$\cos \left(\phi+\frac{\pi}{2}\right):=\frac{y}{\sqrt{x^{2}+y^{2}}}$

which implies

$-\sin \left(\phi+\frac{\pi}{2}\right) \mathrm{d} \phi=\frac{x^{2}}{r^{3}} \mathrm{~d} y$ where $r:=\sqrt{x^{2}+y^{2}}$

leads to

$$
\begin{aligned}
& f(x, \chi)= \\
& \int_{-\frac{\pi}{2}}^{\frac{\pi}{2}} \frac{\cos ^{2} \phi \mathrm{d} \phi}{\sqrt{(x-2 \cos \chi)^{2} \cos ^{2} \phi+(|x| \sin \phi+2 \sin \chi \cos \phi)^{2}}}
\end{aligned}
$$

Equating the denominator of this angular integral to $\sqrt{a-b \sin ^{2}\left(\phi+\frac{\alpha}{2}\right)}$ term by term with respect to the trigonometric functions and solving for the unknown constants $a, b$ and $\alpha$ leaves a sum of complete elliptic integrals of the 1 st and 2nd kind (Gradshtein \& Ryzhik 1994, Ch. 8.1) and

$$
\begin{aligned}
f(x, \chi) & =\frac{1}{\sqrt{a}} \int_{\alpha-\frac{\pi}{2}}^{\alpha+\frac{\pi}{2}} \frac{\cos ^{2}\left(\phi-\frac{\alpha}{2}\right) \mathrm{d} \phi}{\sqrt{1-\frac{b}{a} \sin ^{2} \phi}} \\
& = \begin{cases}K(k)+\frac{E(k)-K(k)}{k} \cos \alpha & k<1 \\
\frac{1}{k} K\left(\frac{1}{k}\right)+\left[E\left(\frac{1}{k}\right)-K\left(\frac{1}{k}\right)\right] \cos \alpha & k>1\end{cases}
\end{aligned}
$$

where

$k:=\sqrt{x^{2}+2 x \cos \chi+1}$ and $\cos \alpha=\frac{1-x \cos \chi}{k}$.

Inserting this result into Eq. (36) leads to a onedimensional integral that has to be evaluated numerically. For symmetrie reasons it suffices to restrict that task to the range $0 \leq \chi \leq \pi / 2$ because $\alpha(m,-\boldsymbol{s})=\alpha^{*}(m, \boldsymbol{s})$ and the integrand is independent of $\operatorname{sgn} \chi$. Therefore:

$$
\begin{aligned}
& \alpha(m, s)= \\
& \int_{-\infty}^{0} \frac{1-\mathrm{e}^{i m s x}}{x^{2}}\left[\frac{1}{k} K\left(\frac{1}{k}\right)+\frac{1-x \cos \chi}{k}\left[E\left(\frac{1}{k}\right)-K\left(\frac{1}{k}\right)\right]\right] \mathrm{d} x \\
& \quad+\int_{0}^{2 \cos \chi} \frac{1-\mathrm{e}^{i m s x}}{x^{2}}\left[K(k)+\frac{1-x \cos \chi}{k^{2}}[E(k)-K(k)]\right] \mathrm{d} x \\
& +\int_{2 \cos \chi}^{\infty} \frac{1-\mathrm{e}^{i m s x}}{x^{2}}\left[\frac{1}{k} K\left(\frac{1}{k}\right)+\frac{1-x \cos \chi}{k}\left[E\left(\frac{1}{k}\right)-K\left(\frac{1}{k}\right)\right]\right] \mathrm{d} x .
\end{aligned}
$$


Apart from logarithmic singularities at $x=0$ and at $x=2 \cos \chi$ where some series expansion of the integrand has to be employed, numeric integration poses no further problem and it is best to store the results on disk for table look-up and interpolation, because this function $\alpha(m, s)$ will be needed for the calculation of $\left\langle I\left(\zeta_{1}\right) I\left(\zeta_{2}\right)\right\rangle$ in every upcoming lensing situation.

In the course of further calculations analytical approximations for the function $\alpha(m, s)$ will be used when $s \rightarrow 0$ or $s \rightarrow \infty$. In case of small $s$ :

$\alpha(m, s) \underset{s \rightarrow 0}{\longrightarrow} \alpha_{0}(m, s):=$

$\pi m^{2} s^{2}\left(\frac{3}{4}+0.6 \cos ^{2} \chi-\frac{1}{2} \ln m s\right)-i \pi m s \cos \chi$

and with large $s$

$$
\begin{aligned}
& \alpha(m, s) \underset{s \rightarrow \infty}{\longrightarrow} \pi m s \\
& -i \pi\left(\frac{m s \cos \chi}{2 \sqrt{1+(m s)^{2}}}+\frac{\sin (2 m s \cos \chi)}{4 m s}+\frac{3 \cos (2 m s \cos \chi)}{4(m s)^{2}}\right) \\
& \approx \pi m s-i \frac{\pi}{2} \cos \chi
\end{aligned}
$$

where the rather complicated expression for the imaginary part has been included to illustrate the decaying oscillation that is present in $\alpha(m, s)$.

To include a mass spectrum in the lens it is easiest to substitute Eq. (34) by the weighted sum

$a(s):=\sum_{i} \frac{n_{i}}{n} \alpha\left(m_{i}, s\right)$

with weight factors $n_{i} / n$ approximating the probability $\phi(m) \mathrm{d} m$. Because of $\alpha(m, \boldsymbol{s}) \equiv \alpha(1, m \boldsymbol{s})$ this can be evaluated without difficulties.

At least for sources with $R>1$ it is a good approximation to use the effective mass (Refsdal \& Stabell 1991)

$m_{\mathrm{eff}}:=\frac{\left\langle m^{2}\right\rangle}{\langle m\rangle}$

instead of the mass spectrum.

To allow for peculiar motion of the stars in this context, Eq. (25) has to be modified accordingly. By substitution of $\boldsymbol{\rho}+\frac{1}{2} \boldsymbol{v}_{\mathrm{s}} t$ for $\boldsymbol{\rho}$ with random star velocity $\boldsymbol{v}_{\mathrm{s}}$ and probability density for the star velocity $\phi\left(\boldsymbol{v}_{\mathrm{s}}\right)$ the expectation value can be calculated for every time difference $t$. As a result the function $a(s)$ becomes time dependent and has to be evaluated on a three dimensional grid, so the numerical effort will be too large for practical applications.

\section{Evaluation of $\left\langle I\left(\zeta_{1}\right) I\left(\zeta_{2}\right)\right\rangle$}

After insertion of Eqs. (23), (24) into Eq. (13) the integration over $\mathrm{d}^{2} R_{\mathrm{c}}$ is resulting in a factor $(2 \pi)^{2} \delta(2 J T)$, where

$J:=J_{0}-\kappa \mathbf{1}$

and $\mathbf{1}$ is representing the $2 \times 2$ unit matrix.
Exploiting this $\delta$-function the $\mathrm{d}^{2} T$ integral is easily evaluated and taking $\zeta:=\zeta_{2}-\zeta_{1}$, Eq. (13) reduces to the fourdimensional integral

$$
\begin{aligned}
& \left\langle I\left(\zeta_{1}\right) I\left(\zeta_{2}\right)\right\rangle=\frac{\mathcal{I}_{0}^{2}}{(4 \pi)^{2}\left|1-\kappa_{\mathrm{c}}\right|^{4} \operatorname{det} J} \\
& \quad \times \int_{\mathcal{R}^{4}} Q(\boldsymbol{t}, \boldsymbol{\rho}) \exp \left(-\frac{R^{2}}{8} t^{2}+\frac{i}{2} \operatorname{sgn} \kappa \boldsymbol{t}^{T}\left(\zeta-J_{0} \rho\right)\right) \mathrm{d}^{2} t \mathrm{~d}^{2} \rho \\
& =\langle I\rangle^{2} \frac{\operatorname{det} J}{(4 \pi)^{2}} \int_{\mathcal{R}^{4}} \mathrm{e}^{-\frac{R^{2}}{8} t^{2}-\frac{n}{2} \rho^{2} a(s)+\frac{i}{2} \operatorname{sgn} \kappa t^{T}\left(\zeta-J_{0} \rho\right)} \mathrm{d}^{2} t \mathrm{~d}^{2} \rho
\end{aligned}
$$

Since the $\mathrm{d}^{2} \rho$ integral is not absolutely convergent with $|\boldsymbol{t}| \rightarrow 0$, convergence will be forced by adding $-\varepsilon \rho^{2} / 2$ to the exponent and taking the limit $\varepsilon \rightarrow 0$ after integration (Deguchi \& Watson 1987). Rewriting the integral Eq. (49) by application of the coordinate transformation Eq. (31) $t \rightarrow s$ and taking $\mathrm{d}^{2} t=\rho^{2} \mathrm{~d}^{2} s$, this function reads

$$
\begin{aligned}
\left\langle I\left(\boldsymbol{\zeta}_{1}\right) I\left(\boldsymbol{\zeta}_{2}\right)\right\rangle & =\langle I\rangle^{2} \frac{\operatorname{det} J}{4 \pi} \lim _{\varepsilon \rightarrow 0} \int_{\mathcal{R}^{2}} h_{\varepsilon}(\boldsymbol{s}, \zeta) \frac{\mathrm{d}^{2} s}{s^{2}} \\
& =\langle I\rangle^{2}\left(H_{0}+\frac{\operatorname{det} J}{4 \pi} H(\boldsymbol{\zeta})\right)
\end{aligned}
$$

where

$H(\zeta):=\int_{\mathcal{R}^{2}} \lim _{\varepsilon \rightarrow 0} h_{\varepsilon}(\boldsymbol{s}, \zeta) \frac{\mathrm{d}^{2} s}{s^{2}}$

and

$h_{\varepsilon}(s, \zeta):=\frac{s^{2}}{4 \pi} \int_{\mathcal{R}^{2}} \exp \left(-\frac{s}{2} \rho^{T} \tilde{A} \boldsymbol{\rho}+\frac{i}{2} s \operatorname{sgn} \kappa \boldsymbol{\rho}^{T} U^{T} \zeta\right) \rho^{2} \mathrm{~d}^{2} \rho$

For abbreviation

$\tilde{A}:=\left(\frac{R^{2}}{4} s+\frac{n}{s} a(s)+\frac{\varepsilon}{s}\right) \mathbf{1}+i \operatorname{sgn} \kappa U^{T} J_{0}$

has been used, together with

$U:=\left(\begin{array}{cc}\cos \chi & -\sin \chi \\ \cos \chi & \sin \chi\end{array}\right)$ and $t=s U \rho$.

The quadratic form $\rho^{T} \tilde{A} \rho$ does only depend on the symmetric part of $\tilde{A}$ so it can be rewritten

$\rho^{T} \tilde{A} \rho=\rho^{T} A \rho$ with $A:=\frac{1}{2}\left(\tilde{A}+\tilde{A}^{T}\right)$.

Since $A$ is independent of $\rho$ the integral Eq. (52) can be transformed to a pair of coupled Gaussians by partial integration and it evaluates to

$h(s, \zeta)=\frac{1}{2 \sqrt{\operatorname{det} A}} \mathrm{e}^{-\frac{s}{8} \zeta^{T} U A^{-1} U^{T} \zeta}\left[\frac{\operatorname{Tr} A}{\operatorname{det} A}-\frac{s}{4} \zeta^{T} U A^{-2} U^{T} \zeta\right]$.

Taking as a shorthand

$b(s):=\frac{R^{2}}{4} s+n \frac{a(s)}{s}+\frac{\varepsilon}{s}+i \operatorname{sgn} \kappa \cos \chi$

the trace and the determinant of $A$ are

$\operatorname{Tr} A=2 b(s)$ and $\operatorname{det} A=b(s)^{2}+\gamma^{2}$ 
respectively and using $\zeta=[\zeta \cos \psi, \zeta \sin \psi]^{T}$ this results in

$$
\begin{aligned}
h(s, \zeta)= & {\left[\frac{b}{\left(b^{2}+\gamma^{2}\right)^{\frac{3}{2}}}-\frac{s \zeta^{2}}{8} \frac{b^{2}-2 i b \gamma \operatorname{sgn} \kappa \cos (\chi-2 \psi)-\gamma^{2}}{\left(b^{2}+\gamma^{2}\right)^{\frac{5}{2}}}\right] } \\
& \times \exp \left(-\frac{s \zeta^{2}}{8} \frac{b-i \gamma \operatorname{sgn} \kappa \cos (\chi-2 \psi)}{\left(b^{2}+\gamma^{2}\right)}\right)
\end{aligned}
$$

Because of $h_{\varepsilon}(-s, \zeta)=h_{\varepsilon}^{*}(s, \zeta)$, the function $H(\zeta)$ is real and can be rewritten

$H(\zeta)=\frac{1}{2} \int_{\mathcal{R}^{2}} \lim _{\varepsilon \rightarrow 0} \mathfrak{R} e\left[h_{\varepsilon}(s, \chi ; \zeta)+h_{\varepsilon}(s,-\chi ; \zeta)\right] \frac{\mathrm{d}^{2} s}{s^{2}}$

For $s_{0}<s<s_{\infty}$ with some cut-off parameters $s_{0}, s_{\infty}$ the angular integral can be evaluated numerically using a Romberg scheme followed by radial integration of the same type. For $s>s_{\infty}, R^{2} s_{\infty} \gg 1$ the approximation $b(s) \approx s R^{2} / 4$ can be used first to evaluate the radial integral analytically and doing the numerical angular integral afterwards. For $s$ below $s_{0} \approx 4 \times 10^{-5} / R^{2}$ the function $h(s, \zeta)$ with decreasing $s$ shows an increasingly violent oscillation near $\cos \chi \approx \gamma /(1-\kappa)$. To gain the necessary numerical accuracy, those peaks have to be tracked with high precision. When this can be no longer achieved as $s \rightarrow 0$, a semianalytical evaluation of the angular integral can be successful. By using the approximation Eq. (44) and taking the first few terms of a series expansion with respect to $\zeta^{2}$, the angular integral can be done analytically if $\gamma=0$. In case of a nonvanishing shear a series expansion with respect to $\gamma$ (or $\gamma^{-1}$ if $\gamma>1$ ) can be used, followed by partial fraction decomposition with respect to $\cos \chi$ to get an acceptible approximation to the integrand. With the final radial integration only a logarithmic singularity at the origin poses some minor numerical difficulty. Severe limitations arise with $\kappa \rightarrow 0$ and $R \ll 1$ when the cut-off parameter $s_{0}$ has to be taken so large, that Eq. (44) is no longer applicable. To overcome this limitation further improvement of the integration technique is needed. In the special cases $\kappa=1 \pm \gamma$ the amplification of a point source becomes infinite and the expected variance drops to zero logarithmically (Deguchi \& Watson 1987). This result can be confirmed by calculations for smaller sources. With large sources $(R>50)$ this dip was confined to small intervalls around $\kappa=1 \pm \gamma$, a region where the numerical calculations became unreliable.

In the remaining part of Eq. (50)

$H_{0}=\frac{\operatorname{det} J}{4 \pi}\left(\lim _{\varepsilon \rightarrow 0} \int_{\mathcal{R}^{2}} h_{\varepsilon}(\boldsymbol{s}, \boldsymbol{\zeta}) \frac{\mathrm{d}^{2} s}{s^{2}}-\int_{\mathcal{R}^{2}} \lim _{\varepsilon \rightarrow 0} h_{\varepsilon}(\boldsymbol{s}, \boldsymbol{\zeta}) \frac{\mathrm{d}^{2} s}{s^{2}}\right)$

only a small region with radius $\delta$ around the origin contributes. With larger $|\boldsymbol{s}|$ the influence from the $\varepsilon$ can be neglected so that

$H_{0}=\frac{\operatorname{det} J}{4 \pi}\left(\lim _{\varepsilon \rightarrow 0} \int_{\mathcal{U}_{\delta}(0)} h_{\varepsilon}(\boldsymbol{s}, \boldsymbol{\zeta}) \frac{\mathrm{d}^{2} s}{s^{2}}-\int_{\mathcal{U}_{\delta}(0)} \lim _{\varepsilon \rightarrow 0} h_{\varepsilon}(\boldsymbol{s}, \boldsymbol{\zeta}) \frac{\mathrm{d}^{2} s}{s^{2}}\right)$

These integrals can be evaluated easily when $\gamma=0, \zeta=0$ and therefore det $J=(1-\kappa)^{2}$. In this case the function $a(s)$ can be approximated with the help of Eq. (44)

$a(s) \underset{s \rightarrow 0}{\longrightarrow}-i \pi\langle m\rangle s \cos \chi+O\left(s^{2}\right)$ and

$h_{\varepsilon}(\boldsymbol{s}, \mathbf{0})=\left(\frac{R^{2}}{4} s+\frac{\varepsilon}{s}+i \operatorname{sgn} \kappa(1-\kappa) \cos \chi\right)^{-2}$.

Angular integration leads to

$$
\int_{0}^{2 \pi} h_{\varepsilon}(\boldsymbol{s}, \mathbf{0}) \mathrm{d} \chi=2 \pi \frac{\frac{R^{2}}{4} s+\frac{\varepsilon}{s}}{\left(\left(\frac{R^{2}}{4} s+\frac{\varepsilon}{s}\right)^{2}+(1-\kappa)^{2}\right)^{\frac{3}{2}}}
$$

and

$$
\begin{gathered}
H_{0}=\frac{\operatorname{det} J}{2} \lim _{\varepsilon \rightarrow 0} \int_{0}^{\delta}\left(\frac{\frac{R^{2}}{4} s^{2}+\varepsilon}{\left(\left(\frac{R^{2}}{4} s^{2}+\varepsilon\right)^{2}+(1-\kappa)^{2}\right)^{\frac{3}{2}}}\right. \\
\left.-\frac{\frac{R^{2}}{4} s^{2}}{\left(\left(\frac{R^{2}}{4} s^{2}\right)^{2}+(1-\kappa)^{2}\right)^{\frac{3}{2}}}\right) s \mathrm{~d} s=\frac{1}{2},
\end{gathered}
$$

which is in perfect agreement with the findings of Deguchi \& Watson (1987), so the final result will be

$\left\langle I\left(\zeta_{1}\right) I\left(\zeta_{2}\right)\right\rangle=\langle I\rangle^{2}\left(\frac{1}{2}+\frac{\operatorname{det} J}{4 \pi} \int_{\mathcal{R}^{2}} \lim _{\varepsilon \rightarrow 0} h_{\varepsilon}(s, \zeta) \frac{\mathrm{d}^{2} s}{s^{2}}\right)$.

The only special case where this right hand integral is analytically solvable occurs with $\zeta=0, \gamma=0$ and a vanishing number of stars $n=0$, which gives the trivial result $\left\langle I^{2}\right\rangle=\langle I\rangle^{2}$ since there is no variability without spatial mass fluctuations. This just serves as a simple but incomplete test for correctness of the result Eqs. (67), (59). More convincing evidence for the capability of the presented method can be gathered by comparison with findings by Refsdal \& Stabell (1991), who estimate the variance of the amplification for large sources with constant surface brightness

$\frac{\left\langle\mu^{2}\right\rangle}{\langle\mu\rangle^{2}}=\frac{4 \kappa}{R^{2}}$

To derieve the result for a Gaussian brightness profile from this, several large sources can be added together, each weighed with the local brightness. If this sum is then approximated by a surface integral, the variance of the flux density comes out to be

$\frac{\left\langle I^{2}\right\rangle}{\langle I\rangle^{2}}=\frac{2 \kappa}{R^{2}}$

which is half as large as in the case of constant surface brightness with source radius $R$. This is in stark contrast to the findings of Seitz et al. (1994) who obtain a variance that is twice as large. Their correction factor to the Refsdal, Stabell result Eq. (68) did not lead to an improvement in this context.

Numerical calculations confirm the result Eq. (69) with $20 \leq R \leq 100$. When $R \approx 100$, the variance drops below $10^{-5}$, the numerical precision becomes important and the results are about 5\% larger than Eq. (69) predicts. For even larger sources the numerical calculations become completely unreliable. 


\section{Applications}

If a macroscopic lens model for a multiple quasar has been determined from observations, the local mass density $\kappa_{\mathrm{t}}$ and the shear $\gamma^{\prime}$ at the image positions can be calculated from the gravitational potential of the lens. The variance imprinted on the light curve of each macroimage by microlensing

$\operatorname{Var} I:=\left\langle I^{2}\right\rangle-\langle I\rangle^{2}$

is then completely determined by the mass densities $\kappa_{\mathrm{S}}$ in stars $^{1}$ and other compact objects, the corresponding mass spectrum $\phi(m)$ introduced with Eq. (19) and the source radius $R$ which, of course has to be equal in the different macroimages of the same source. If, as will be assumed from now on, the mass spectrum can be approximated by an effective mass Eq. (47), its value can be taken as the unit mass used with the definition of the Einstein radius Eq. (3).

Equation (67) can then be used with $\boldsymbol{\zeta}_{1}=\boldsymbol{\zeta}_{2}$ to determine the source radius as a function of variance and normalized mass density $\kappa$, where the variance has to be estimated from measured light curves. Although straightforward in principle this is not an easy task to accomplish because variability intrinsic to the source and measurement errors add to the variance of an observed quasar image. Intrinsic variability can be accounted for by determination of the crosscorrelation between any two light curves from different macroimages of the same source that have been shifted with respect to each other by their relative time delay. With this approach microlensing variability cancels at least with an infinite light curve, because it is statistically independent for different macroimages. Additional variance from statistical noise will be uncorrelated in time and therefore show up as a discontinuity of the autocorrelation function at zero time lag. When those additional sources of variance are accounted for, the remaining estimate for microlensing variance can be used to determine the source radius $R$ as a function of mass density. Irregular sampling of the data can still provide a source of error, if brightness changes are missed by the observations, as has been shown by Wambsganss (1992).

Whereas the calculation of the variance supplies just one number per macroimage, more information can be extracted from the autocorrelation function for microlensing

$q\left(\boldsymbol{\zeta}_{2}-\boldsymbol{\zeta}_{1}\right):=\frac{\left\langle I\left(\boldsymbol{\zeta}_{1}\right) I\left(\boldsymbol{\zeta}_{2}\right)\right\rangle-\langle I\rangle^{2}}{\left\langle I^{2}\right\rangle-\langle I\rangle^{2}}$

According to Eqs. (59), (67) this is a function of the normalized lens parameters $\kappa$ and $\gamma$, the source size $R$, the angle $\psi$ between the direction of movement and the direction of the shear if $\gamma \neq 0$ and of the effective transverse velocity via the transformation to the time domain $\zeta=\boldsymbol{V} t$

$\boldsymbol{V}=\frac{\boldsymbol{v}_{\mathrm{s}}}{1+z_{\mathrm{s}}}-\frac{\boldsymbol{v}_{\mathrm{d}}}{1+z_{\mathrm{d}}} \frac{D_{\mathrm{s}}}{D_{\mathrm{d}}}+\frac{\boldsymbol{v}_{\mathrm{o}}}{1+z_{\mathrm{d}}} \frac{D_{\mathrm{ds}}}{D_{\mathrm{d}}}$

derieved by Kayser et al. (1986) for cosmologies with no space curvature (Miralda-Escudé 1991). To characterize an autocorrelation function the correlation time $t_{h}$ or the correlation length $\zeta_{h}=V t_{h}$, defined by $q\left(\zeta_{h}\right)=1 / 2$ are convenient but they are not sufficient to describe the autocorrelation function completely.

\footnotetext{
${ }^{1} \kappa_{\mathrm{c}}$ can be calculated from $\kappa_{\mathrm{c}}=\kappa_{\mathrm{t}}-\kappa_{\mathrm{s}}$.
}

The actual (dis-)agreement between a microlensing model and an empirical autocorrelation function $f(t)$ can be expressed as

$$
\|f(t)-q(\boldsymbol{V} t)\|_{p}:=\left(\int_{0}^{T_{\max }} w(t)[f(t)-q(\boldsymbol{V} t)]^{p} \mathrm{~d} t\right)^{1 / p}
$$

with weight factor $w(t)$ reflecting the statistical reliability for the empirical function $f(t)$, where using $w(t) \equiv 1$ the area between the two functions ( $p=1)$, the conventional Euclidian norm $(p=2)$ and the Chebychev (maximum) norm $(p=\infty)$ have been considered for test calculations. With increasing $p$ more weight is given to data showing large differences between the two functions. Since all autocorrelation functions are normalized to unity at the origin, those large differences preferably occur at large time lags where the reliability of the empirical autocorrelation function $f(t)$ is worst. Minimization of $\|f(t)-q(\boldsymbol{V} t)\|_{p}$ then results in larger differences near the origin, favouring best approximations, which are quite different qualitatively as can be seen for example by comparison of their derivatives at $t_{h}$. This effect can be compensated for by forcing both functions $f$ and $q$ to agree near the origin. Using approximations by truncated series in $-t^{2}$ and $-\zeta^{2}$ respectively, an appropriate choice of the velocity parameter $V$ forces equality of the curvature near the origin for any pair of autocorrelation functions. In this way differences between the functions will arise only for larger time lags and the choice of norm will no longer be that crucial but might still lead to slightly different results.

Searching for the best approximation to an empirical autocorrelation function in five dimensional parameter space includes a large number of function evaluations, which need cputime in the order of minutes for every set of arguments $\kappa$, $\gamma, R, \psi$ and $\zeta$. This effort can be reduced if $\kappa_{\mathrm{t}}$ and $\gamma^{\prime}$ are known from a macrolens model and only $\kappa_{\mathrm{c}}$ is left as a free parameter to fix $\kappa$ and $\gamma$. But, as Wambsganss \& Paczyński (1994) pointed out, the gravitational potential in the lens cannot be uniquely determined from the image positions alone and additional knowledge about the time delay or the flux ratios is needed, a condition that is not necessarily fullfilled for multiple quasars.

Instead of determining the velocity parameter $V$ by the aforementioned method, for every set of parameters $\kappa, \gamma, R$ and $\psi$, it can be searched for by minimizing the norm $\| f(t)-$ $q(\boldsymbol{V} t) \|_{p}$ with respect to $V$. Several test calculations led to only minor changes or none at all in the resulting best approximations, but clearly reduced $\|f(t)-q(\boldsymbol{V} t)\|_{p}$ with other model parameters, especially with more devious choices.

Whereas the use of the autocorrelation function reduces systematic errors because of normalization by the variance, the evaluation of statistical errors becomes more involved, since $\left\langle I\left(t_{1}\right) I\left(t_{2}\right)\right\rangle$ and Var $I$ are statistically dependent and error estimation for their ratio is not trivial.

\section{6. $B 1600+434$, a test case}

To get an impression of the capability of the presented method, the VLA $8.4 \mathrm{GHz}$ radio data of the double quasar B1600+434 
(Koopmans et al. 2000) have been analyzed. The light curves consisting of 75 data points span 243 days and show large uncorrelated variability in addition to a negative linear trend. Six data points they flagged as outliers have not been considered in the upcoming analysis. Koopmans \& de Bruyn (2000) examined the fluctuations with respect to the running mean and discussed scintillation and microlensing by superluminal motion in a radio jet (Gopal-Krishna \& Subramanian 1991) as a possible cause. They found evidence that supported the latter and allowed for the deduction of information about the source so it was challenging to reexamine the data using an independent method.

Straightforward estimation of the autocorrelation function by the Edelson-Krolic method (1988) does not lead to stable statistical results because of too few data points available and the estimate strongly depending on bin size. Extension of the light curve to two data points per day by linear interpolation results in a continuous autocorrelation function that can be used for comparison with model calculations. This proceedure is not suitable for practical purposes since it can introduce uncontrollable bias and does not supply any means for error estimation, but because of its simplicity it is almost ideal as a test case. In the following $w(t) \equiv 1$ has been used in Eq. (73).

B1600+434 is a $z=1.59$ double quasar with image separation 1.39 arcsec. The lens was identified as an edge-on spiral galaxy at $z=0.41$ (Jackson et al. 1985; Fassnacht \& Cohen 1988). Following Koopmans and de Bruyn (2000) and based on the singular isothermal mass distribution found by Koopmans et al. (1998), the normalized mass density and shear are $\kappa_{\mathrm{t}}=\gamma^{\prime}=0.2$ at image A which is seen though the galaxie's halo and $\kappa_{\mathrm{t}}=\gamma^{\prime}=0.9$ at image B near the center of the lens galay.

At image A approximation by microlensing model functions with a $T_{\max }$ of up to six days has been possible. Because of oscillations in the empirical autocorrelation functions no qualitative agreement with model functions has been found for larger $T_{\max }$. At image B approximations for $T_{\max } \leq 10 \mathrm{~d}$ were possible. No reason for these oscillations has been found yet. Similar oscillations show up with the autocorrelation functions to the $8.5 \mathrm{GHz}$ VLA data of the quadruple lens system B1608+656 (Fassnacht et al. 1999).

Model autocorrelation functions have been calculated for sources with $R=0.5,0.7,1,2,3$ and 5 Einstein radii, angles between the direction of motion and the shear $0 \leq \psi \leq \pi / 2$ and different values of $\kappa_{\mathrm{c}}$. Figure 2 displays contourplots for the difference $\|f(t)-q(\boldsymbol{V} t)\|_{2}$ produced by twodimensional interpolation. The ratio of $R / V$ is only slowly varying across the parameter space and the gradient of $\|f(t)-q(\boldsymbol{V} t)\|_{2}$ is minimal approximately along the lines $\cos \psi \approx R$ constant. The resulting bestapproximations are shown in Fig. 1. The large differences in estimated radii and velocities from both images stem from a large value for $\kappa_{\mathrm{c}}$ in image B. If the resulting factor $\sqrt{\left|1-\kappa_{\mathrm{c}}\right|}=0.4$ is accounted for, the ratios of the physical quantities become $r_{A} / r_{B}=0.875$ and $v_{A} / v_{B}=1.25$ respectively. Allowing for scatter broadening of image B near the galaxie's disk and assuming the effective mass to be larger at that image reduces these discrepancies but quantitative results have to be based on a more appropriate data analysis. But even then it is

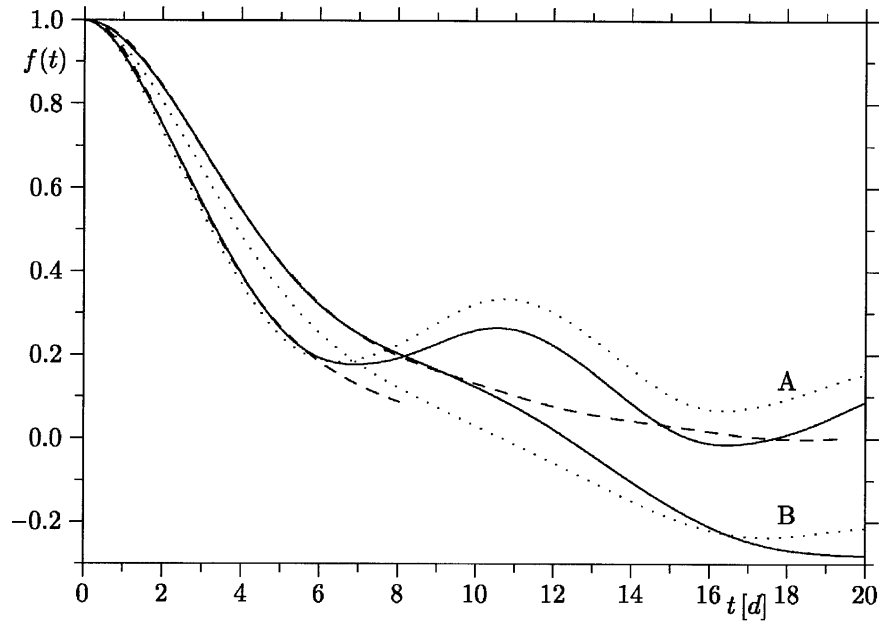

Fig. 1. Autocorrelation functions of B1600+434A,B from interpolated light curves with six outliers ignored (solid), complete record (dotted) and model calculations with $\kappa_{\mathrm{c}}=0, R=0.7 \zeta_{0}, V=0.5 \zeta_{0} / \mathrm{d}$ at image $\mathrm{A}$ and $\kappa_{\mathrm{c}}=0.84, R=2.0 \zeta_{0}, V=1.0 \zeta_{0} / \mathrm{d}$ at image B (dashed), each with $\psi=18^{\circ}$.
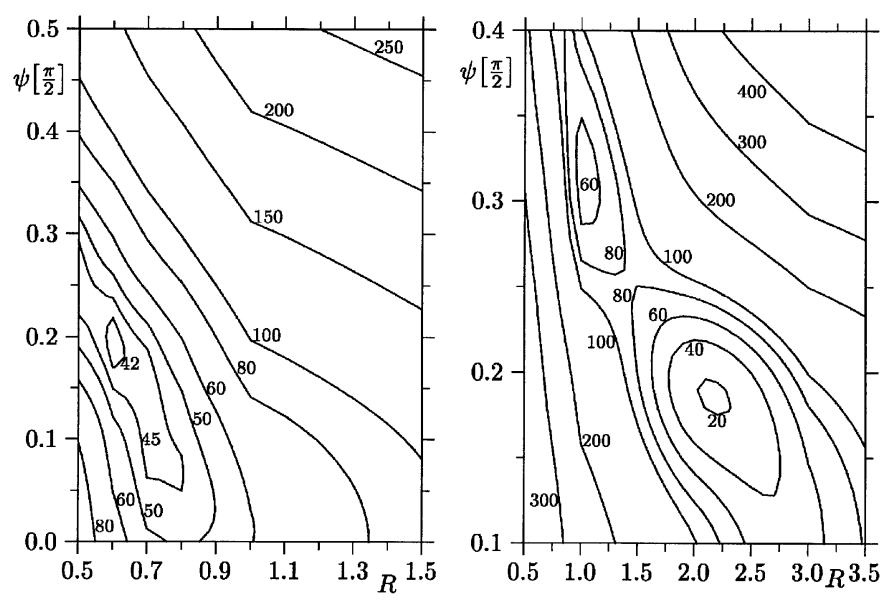

Fig. 2. B $1600+434 \mathrm{~A}, \mathrm{~B}$ : contourlines of $10^{3} \cdot\|f(t)-q(\boldsymbol{V} t)\|_{2}$ for $T_{\max }=$ $6 \mathrm{~d}$ at image A (left, $\kappa_{\mathrm{c}}=0$ ) and $T_{\max }=10 \mathrm{~d}$ at B (right, $\kappa_{\mathrm{c}}=0.84$ ).

not possible to determine the absolute values for $m_{\text {eff }}$ because all results are derieved from normalized quantities that do not depend on the Einstein radius' value and therefore are independent of a physical length scale.

The variance ratio resulting from the best approximating model $\operatorname{Var} I_{A} / \operatorname{Var} I_{B}=3.0$ is only $5 \%$ larger than the ratio estimated from the data, a finding that so far confirms the reliability of the presented methods. The variances themselves differ considerably. Whereas estimation from data led to $\operatorname{Var} I_{A}=6.1 \times 10^{-4}$, modelling resulted in $\operatorname{Var} I_{A}=0.198$. Following Koopmans \& de Bruyn (2000) and assuming that only a fraction $f$ of the total radiation is emitted by the radiojet, the variance of the jet alone is by a factor $1 / f^{2}$ larger than the variance of the whole source. Comparison of the variance estimated from data and the model calculations results in $f=5.5 \%$, a value that agrees well with the Koopmans and de Bruyn estimate of $5 \% \leq f \leq 11 \%$. 


\section{Conclusions}

On the basis of the theory of gravitational lensing a probability theoretical procedure has been developed, that opens up the possibility to analyze the effect of microlensing on images of a multiple quasar. For a statistical investigation of a photometric series of observations the variance and the autocorrelation function of the light curve are suitable. While so far it was necessary to produce a large number of artificial light curves by means of numerical proceedures like ray shooting that formed the basis for statistical model calculations, the probabilistic procedure presented here enables a direct numeric computation of autocorrelation function and variance. Thus it can be ensured that the achieved precision is limited only by the numerics and the computed model functions are free of otherwise unavoidable statistical errors. These limitations become important with $R \ll 1$ when the numerical accuracy is no longer sufficient. Further improvement of the algorithms is essential.

The $8.5 \mathrm{GHz}$ VLA radio data of the double quasar B1600+434 obtained by Koopmans et al. (2000) have been used to test the probabilistic method presented here. According to Koopmans \& de Bruyn (2000) the variability of the difference light curve is due to microlensing of a superluminal jet component. Because of the large apparent transverse velocity of the source peculiar motion of individual stars can be neglected and the lens can be taken as static. Although variability on time scales of less than three days remains almost unobserved, a crude estimate of the autocorrelation functions allows for consistent approximations by model functions. The system parameters inferred by this comparison agree quite well with the findings of Koopmans and de Bruyn. The discovery of a radio loud lens system with rapid external variability raises hope for further detection of microlensing in radio loud multiple quasars. Since light curves of multiple quasars are needed anyway (determination of the Hubble constant $H_{0}$ f. ex.) there is no extra effort needed to get light curves, which exhibit also an observation density sufficient for the computation of autocorrelation functions. In contrast to lens system observed in the optical where several decades of regular observations are needed, light curve lengths of one year can be sufficient for the analysis by this probabilistic method. A main problem will be the development of a search strategy for best parameter combination that does better than systematic calculations on a parameter grid.

A major task that still has to be accomplished is the estimation of an autocorrelation function from data. While interpolation of a light curve can introduce bias in an uncontrollable way, the Edelson-Krolic (1988) method leads to highly discontinuous correlation functions when small bins are used. With larger bins too much information from a light curve will get lost. The minimum dispersion method developed by Pelt (1983) tackles this problem in a different way but has not been tested yet in the context of autocorrelation function determination.

As an alternative approach the estimation of the periodogram from the Fourier transformed data is common. In this way the estimates for large time lags can be improved because all data are used for each estimate. Comparison with model calculations can be accomplished either by back transformation of the periodogram to the time domain or by Fourier transforming Eqs. (59), (67) with respect to time. A characteristic all these periodogram methods have in common is the fact that they are direct estimates that are inconsistent, meaning that the expected error does not decrease with increasing light curve length (Mullis \& Scharf 1991; Thomson 1982).

A standard way to overcome this problem would be the subdivision of the light curve in question into several eventually partly overlapping subsections, that are then evaluated independently and to take the average of these results. In the case of multiple quasars light curves are still too short to get sufficient information by averaging individual light curve sections. Thomson (1982) describes a nonparametric procedure that estimates the spectrum by using projection operators. He explicitly stated that this procedure is suitable for small data sets, and not based on a theory that is dependent on infinite light curves. Such a method then opens up the possibility to obtain statistical information even from short light curves or from lens systems with small effective transverse velocity like the double quasar QSO 0957+961 for example.

What remains to be clariefied is in what respect a separation of microlens effect and intrinsic variability can be obtained in Fourier space, or whether the comparison with model calculations must still be accomplished in the time representation, and only the computation of the empirical correlation functions can be replaced by stable procedures.

It is planned to investigate this topic further, since the investigation of autocorrelation functions promises to be an ideal means for the deduction of complete statistical models for multiple lens systems.

\section{References}

Chandrasekhar, S. 1943, Rev. Mod. Phys., 15, 1

Deguchi, S., \& Watson, W. D. 1987, Phys. Rev. Lett., 59(24), 2814

Edelson, R. A., \& Krolik, J. H. 1988, ApJ, 333, 646

Fassnacht, C. D., \& Cohen, J. G. 1988, AJ, 115, 377

Fassnacht, C. D., Pearson, T. J., \& Readhead, A. C. S. 1999, ApJ, 527, 498

Gopal-Krishna, \& Subramanian, K., Nature, 349, 766

Gradshtein, I., \& Ryzhik, I. 1994, Tables of Series, Products and Integrals (Academic Press)

Grieger, B., Kayser, R., \& Refsdal, S. 1988, A\&A, 194, 54

Grieger, B., Kayser, R., \& Scramm, T. 1991, A\&A, 252, 508

Jackson, N., de Bruyn, A. G., Myers, S., et al. 1995, MNRAS, 274, 25

Kayser, R., Refsdal, S., \& Stabell, R. 1986, A\&A, 166, 36

Koopmans, L. V. E., de Bruyn, A. G., \& Jackson, N. 1998, MNRAS, 295,534

Koopmans, L. V. E., de Bruyn, A. G., Xanthopoulos, E., \& Fassnacht, C. D. 2000, A\&A, 356, 391

Koopmans, L. V. E., \& de Bruyn, A. G. 2000, A\&A, 358, 793

Kundić, T., \& Wambsganss, J. 1993, ApJ, 404, 455

Lewis, G. F., Miralda-Escudé, J., Richardson, D. C., \& Wambsganss, J. 1993, MNRAS, 261, 647

Miralda-Escudé, J. 1991, The magnification of stars crossing a caustic. I - Lenses with smooth potentials, ApJ, 379, 94

Mullis, C. T., \& Scharf, L. L. 1991, in Advances in spectrum analysis and array processing, vol. 1, ed. S. Haykin (Prentice-Hall)

Paczyński, B. 1986, ApJ, 301, 2 
Pelt, J. 1983, ESA Spec. Publ., ESA SP 201, 37

Refsdal, S., \& Stabell, R. 1991, A\&A, 250, 62

Refsdal, S., \& Stabell, R. 1993, A\&A, 278, L5

Refsdal, S., \& Stabell, R. 1997, A\&A, 325, 877

Refsdal, S., Stabell, R., Pelt, J., \& Schild, R. 2000, A\&A, 360, 10

Schneider, P., Ehlers, J., \& Falco, E. E. 1992, Gravitational Lenses (Springer)

Schneider, P., \& Weiss, A. 1986, A\&A, 164, 237

Schmidt, R., \& Wambsganss, J. 1998, A\&A, 335, 379

Seitz, C., \& Schneider, P. 1994, A\&A, 288, 1

Seitz, C., Wambsganss, J., \& Schneider, P. 1994, A\&A, 309, 59

Shalyapin, V. N. 2001, Caustic Crossing in the Gravitational Lens Q2237+0305, AL 27(3), 150

Thomson, D. J. 1982, Spectrum Estimation and Harmonic Analysis, Proc. IEEE, 70, 1055
Wambsganss, J. 1990, Gravitational Microlensing, report MPA 550 Garching

Wambsganss, J. 1992, ApJ, 392, 424

Wambsganss, J., \& Paczyński, B. 1990, AJ, 108, 1156

Wambsganss, J., Paczyński, B., \& Katz, N. 1990, ApJ, 352, 407

Witt, H. J. 1993, ApJ, 403, 530

Witt, H. J., Kayser, R., \& Refsdal, S. 1993, A\&A, 268, 501

Woźniak, P., Alard, C., Udalski, A., et al. 2000, ApJ, 529, 88

Wyithe, J. S. B., Webster, R. L., \& Turner, E. L. 2000a, MNRAS, 312, 843

Wyithe, J. S. B., Webster, R. L., \& Turner, E. L. 2000b, MNRAS, 318, 762

Yonehara, A. 2001, ApJ, 548, L127 